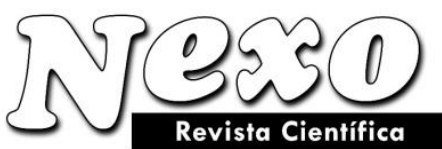

ISSN-E 1995-9516

Universidad Nacional de Ingeniería COPYRIGHT @ (UNI). TODOS LOS DERECHOS RESERVADOS

http://revistas.uni.edu.ni/index.php/Nexo https://doi.org/10.5377/nexo.v34i06.13119

Vol. 36, No. 06, pp. 1589-1598/Diciembre 2021

\title{
Diseño de un proceso industrial para el aprovechamiento continuo de viruta de cuero del proceso de curtido como materia prima en la elaboración de un producto reciclado
}

\section{Design of an industrial process for the continuous use of leather chips from the tanning process as raw material in the elaboration of a recycled product}

\author{
M. F. Zúñiga Nuñez*, A. R. Velando Mosco, J. Villegas Alvarez, J.A. Aguilar Franco \\ Universidad Católica San Pablo. Instituto de Energía y Medio Ambiente. Arequipa, Perú. \\ *mfzuniga@ucsp.edu.pe
}

(recibido/received: 22-septiembre-2021; aceptado/accepted: 28-octubre-2021)

\section{RESUMEN}

Un proceso productivo fue diseñado para el aprovechamiento continuo de viruta de cuero del proceso de curtido para ser utilizado como materia prima para elaborar un producto reciclado (cuero reconstituido). Su objetivo es reutilizar de forma sostenible el residuo de viruta de cuero del proceso de curtido sin generar nuevos residuos sólidos, ya que los potenciales residuos sólidos generados podrán ser nuevamente utilizados en el proceso productivo diseñado. Este proceso está dividido en tres unidades, la primera de acondicionamiento de la materia prima que proporciona las características a la viruta de cuero para ser procesada; la segunda de mezclado y moldeado que mezcla viruta de cuero, agua, resina y; la tercera de prensado y secado que proporciona las propiedades físicas y mecánica para la plancha desarrollada. Esta propuesta permite obtener un proceso ecoamigable ya que va a permitir el uso de viruta de cuero como materia prima elaborar cuero reconstituido; así mismo identificar y definir los parámetros de operación, así como las composiciones respectivas de viruta, agua y resina para la formación de la plancha. Los resultados preliminares evidencian el uso potencial de la viruta de cuero como materia prima para cuero reconstituido sin generar impactos negativos en el ambiente.

Palabras claves: Proceso de curtido, viruta de cuero, cuero reconstituido, uso continuo de residuos sólidos.

\begin{abstract}
A production process was designed for the continuous use of leather shavings from the tanning process to be used as raw material to make a recycled product (reconstituted leather). Its objective is to reuse in a sustainable way the waste of leather shavings from the tanning process without generating new solid waste, since the potential solid waste generated can be used again in the designed production process. This process is divided into three units, the first unit is for the conditioning of the raw material that provides the characteristics to the leather shavings to be processed; the second unit of kneading and molding that mixes shavings of leather, water, resin and; the third third pressing and drying unit that provides the physical and mechanical properties of the developed plate. This proposal allows to obtain an ecological process that will allow the use of leather shavings as raw material for the elaboration of reconstituted leathers; also identify and define the operating parameters, as well as the respective chip, water and resin compositions for the
\end{abstract}


formation of the plaque. Preliminary results show the potential use of leather shavings as raw material to make reconstituted leather without generating negative impacts on the environment.

Keywords: Tanning process, leather shavings, reconstituted leather, continuous use of solid waste.

\section{INTRODUCCIÓN}

Las curtiembres son empresas que se encargan de transformar las pieles de res en materiales imputrescibles, flexibles y duraderos a través de procesos de curtición, sin embargo, este proceso productivo genera gran cantidad de residuos sólidos y efluentes. Los residuos sólidos provienen de la operación de rebajado (igualación del espesor del cuero húmedo o wet blue), luego del proceso de curtición con sales básicas de cromo (Cantera \& Bertola, 2000); asimismo representan alrededor del $40 \%$ de la materia prima y están conformados por carnazas, gamuzón, pelos, restos de carne y viruta de cuero. Sin embargo, se consideran componentes nocivos ya que pueden contener metales y materia orgánica, de clase A3.9 y A3.11. (Medina et al., 2018); es por ello, que según la Ley $\mathrm{N}^{\circ} 27314$ deben ser dispuestos a rellenos de seguridad u otros sistemas debidamente aprobados por la Autoridad de Salud de nivel nacional a través de los servicios de una EPS-RS. Las curtiembres en Arequipa, con el objetivo de cumplir con las disposiciones gubernamentales, invierten recursos (horashombre, almacenes y equipos) y representativas sumas de dinero para una correcta disposición de sus residuos sólidos desaprovechado la oportunidad de emplear dichos desperdicios como materia prima para obtener cuero regenerado de alta calidad.

Actualmente, hay muchas investigaciones y patentes que proponen procesos para reaprovechar las virutas de cuero. (Parker \& Wayme, 1966) desarrollaron una patente para obtener cuero reconstituido mediante las actividades de: dilución de látex poliuretano, formulación de mezcla semilíquida, formación de la hoja, secado de la hoja y curado. Finalmente obtuvieron se obtuvo la formulación base para el cuero regenerado, donde el contenido total de sólidos representa aproximadamente de $10 \%$ a $30 \%$ basado en el peso total de la mezcla de fibra de viruta de cuero, sólidos de látex y agua; y una concentración de mezcla de látex de $2 \%$ a $3 \%$ en peso. Por otro lado, (Ávalos,2013) desarrolló un trabajo de investigación donde aprovechó la viruta de cuero proveniente del proceso de rebajado. Las actividades del proceso abarcan desde la etapa de Molienda y tamizado hasta la etapa de Control de calidad para la obtención del producto; a partir de esta investigación se obtuvo: la caracterización de la viruta de cuero; la formulación base para el cuero regenerado (83\% de viruta de cuero, $10 \%$ de agua y $7 \%$ de Copolímero); el requerimiento de reactivos y equipos; y finalmente las especificaciones del cuero regenerado: espesor de 2 a $5 \mathrm{~mm}$, dimensión de 1 x 1,2 m, 2 cara pulidas, densidad de $450 \mathrm{gr} / \mathrm{m} 2$ y una resistencia al desgarre de 400 a $900 \mathrm{~N} / \mathrm{m} 2$.

Adicionalmente, (Addie \& Karpik, 2011) elaboraron un trabajo de investigación de un procedimiento seco (material con $25 \%$ de humedad en peso) para la formación de cuero reconstituido a partir de virutas de cuero, con cualidades parecidas al cuero convencional. Las actividades del proceso abarcan desde el granulado hasta el prensando e involucran las actividades de: granulado, refinado, licuado, formado, horneado y prensado; obteniendo finalmente el cuero reconstituido. En otro artículo de investigación (Aramayo,2015) presentó un diseño para obtener cuero reconstituido a partir de los residuos generados en las curtiembres; estableciendo parámetros operativos que permitan dar propiedades mecánicas que sean igual o superiores a las del cuero real. Las actividades del proceso abarcan desde la etapa de Desfibrado pasando por las etapas de Mezclado, Filtrado, Secado hasta la etapa de Prensado para la obtención del producto final. Como resultado, se obtuvo una plancha de cuero reconstituido con resistencia a la tracción $(350.91 \mathrm{~N} / \mathrm{cm} 2)$, resistencia al desgarre $(161,45 \mathrm{~N} / \mathrm{cm})$, absorción de agua de $52.27 \%$ y desplazamiento del $31,07 \%$.

(Sánchez y Cortes, 2016) en su investigación, evaluó la alternativa de fabricar cuero reconstituido a partir de viruta del proceso de rebajado, para ello estableció la composición de la viruta revisión bibliográfica para finalmente obtener cuero reconstituido. La viruta de 1 a $3 \mathrm{~mm}$ de granulometría, con 4,3 a 4,5\% de Cr2O3, $14 \%$ de nitrógeno total Kejdahl y de 50 a $60 \%$ de humedad (Alberto et al., 2008) se transformó en cuero reconstituido con $73 \%$ fibras de cuero, $14 \%$ de resinas, $12 \%$ de humedad y $1 \%$ de otros materiales (lubricantes, 
colorantes, entre otros). Asimismo, el autor propuso dos tipos de procesamiento: el proceso en Batch, que produce chapas de 1,0 x 1,2 metros y el procesamiento continuo, que origina el material en rollos de 1,0 metros de ancho; y estableció que dependiendo del proceso de fabricación es posible obtener láminas de cuero reconstituido, con diferentes espesores y que pueden ser sometidas a diversos procesos de acabado, posibilitando la obtención de productos sostenibles para variadas aplicaciones, desempeño diferenciado y costos.

Finalmente, (Azambuja, 2007) desarrolló una invención que posibilita la utilización del material descartado en la producción de cuero; principalmente desechos (viruta de cuero) o recortes de cuero, para obtener un producto que tenga todas las características del cuero original o preferentemente mejorándolas. El proceso consistió en las siguientes etapas: baño enzimático, remoción, desfibración mecánica, formación de manta, prensado en frio, prensado al caliente y acabado. Como resultado se obtuvo una manta de cuero reconstituido con: aspecto visual y sensación táctil similar al cuero natural, resistencia igual o mayor al cizallamiento (al rasgón) que, en el cuero natural, capacidad de ser sometido a los mismos procesos de acabado del cuero natural, propiedades biodegradables.

Por lo expuesto, el presente trabajo de investigación involucra el diseño de un proceso de producción para el aprovechamiento del residuo sólido (viruta de cuero); en términos de utilización de materia prima, considerando los insumos, la maquinaria, los parámetros de control y las principales características de producto obtenido.

\section{METODOLOGÍA}

La metodología experimental propuesta para el diseño de un proceso productivo que aproveche el residuo sólido (viruta de cuero) como materia prima para la obtención de cuero reconstituido en las curtiembres de Arequipa, inició con la etapa de análisis situacional en la que se recabaron datos generales y específicos sobre la gestión (tratamiento, disposición, manejo y/o almacenamiento) de residuos sólidos en las empresas del sector curtiembre, así como la normativa vigente aplicada a las mismas.

Como segunda etapa, se realizó la recopilación de información a través de estudios, artículos, investigaciones, entre otros; que evidencien el uso de viruta de cuero en la elaboración de planchas de cuero. Adicionalmente, como parte de esta etapa, se caracterizó la viruta de cuero, analizando los parámetros más relevantes: potencial hidrógeno $(\mathrm{pH})$, humedad, cromo, aceites y grasas totales de la viruta de cuero. Para ello se realizó un muestreo al azar utilizando un sacabocado (cilindros de hierro o de latón de diferente diámetro, utilizados para perforar y extraer muestras), se recogió un total de 10 muestras de $100 \mathrm{gr}$. cada una, tomadas cada $50 \mathrm{~cm}$. en la pila de virutas de cuero para enviadas a un laboratorio acreditado por el Instituto Nacional de Calidad (INACAL). La información obtenida fue recopilada, procesada y analizada, permitiendo reconocer las etapas que conformaron el proceso productivo y los posibles resultados a obtenerse.

En la tercera etapa se diseñó del proceso para el aprovechamiento de la viruta de cuero y la obtención de cuero reconstituido como producto final, considerando la viabilidad técnica de cada equipo, la viabilidad económica del proyecto, la productividad y rentabilidad del proceso propuesto. Para poder identificar las unidades y etapas del proceso; así como los equipos y protocolos necesarios para su funcionamiento se determinó como primera instancia el artículo final a elaborarse de acuerdo a las características de la plancha de cuero reconstituido a elaborarse, es así que se empleó un matriz para evaluar los siguientes artículos como potenciales productos finales a obtener con el proceso productivo propuesto: relleno de bolsos y carteras, plantillas, cartapacios, contrafuerte de zapatos, cinturones y forro de cuadernos y agendas. Los criterios y las ponderaciones para poder evaluar la potencialidad de fabricación de cada artículo se exponen en la Tabla 1. 
Tabla 1: Matriz de evaluación de artículo

\begin{tabular}{|c|c|c|c|c|}
\hline \multirow[b]{2}{*}{ ARTÍCULO } & \multicolumn{4}{|l|}{ FACTORES } \\
\hline & $\begin{array}{c}\text { Presencia en } \\
\text { el mercado }\end{array}$ & $\begin{array}{c}\text { Precio } \\
\text { de venta }\end{array}$ & $\begin{array}{c}\text { Grosor } \\
\text { requerido }\end{array}$ & Total \\
\hline Peso por factor & 0.3 & 0.5 & 0.2 & 1 \\
\hline $\begin{array}{c}\text { Relleno de } \\
\text { bolsas y carteras }\end{array}$ & 2 & 1 & 3 & 1.7 \\
\hline Plantillas & 4 & 5 & 5 & 4.7 \\
\hline Cinturones & 4 & 5 & 5 & 4.7 \\
\hline $\begin{array}{l}\text { Forros para } \\
\text { cuadernos y } \\
\text { agendas }\end{array}$ & 3 & 2 & 5 & 2.9 \\
\hline Cartapacios & 4 & 4 & 5 & 4.2 \\
\hline $\begin{array}{c}\text { Contrafuerte de } \\
\text { zapatos }\end{array}$ & 3 & 4 & 5 & 3.9 \\
\hline
\end{tabular}

Una vez que se determinó el tipo artículo a fabricar fue posible identificar las unidades, etapas y actividades básicas que debería tener el proceso para la producción de cuero reconstituido. El proceso abarca las siguientes unidades:

\subsection{Primera Unidad - Acondicionamiento de materia prima}

La presente etapa abarca desde la recopilación de la viruta de cuero del proceso de curtido hasta la recepción y pesaje en el proceso productivo. Inicialmente la viruta de cuero será acopiada y colocada en sacos, para posteriormente ser almacenada en un ambiente no expuesto al sol ya que se puede alterar sus características con respecto a la humedad. En caso que la humedad se encuentre por encima de lo establecido (mayor al 60\%) la viruta de cuero tendrá una mayor resistencia al desgarre; mientras que si se encuentra por debajo de lo establecido (menor al 50\%) adquiere una textura de arenilla donde tendrá una menor resistencia al desgarre, un mayor porcentaje de alargamiento a la rotura y la cohesión de las fibras en la etapa de prensado será impedida. En caso la humedad se encuentre por encima de lo establecido, se debe aplicar un proceso de secado previo (dejar al ambiente o utilizar un secador industrial), y si se encuentra por debajo de lo establecido se puede incrementar la humedad mediante un sistema de humidificación industrial hasta alcanzar el porcentaje deseado. Una vez que se inicia el proceso productivo, se pesa la cantidad requerida de viruta para el lote a fabricarse y se llevan los sacos a la zona de molienda. Se mezclan 11,875 1 de agua por cada kilogramo de viruta en un molino de cuchillas con tamiz interno (el tamiz sirve para regular el tamaño del producto procesado) y se obtiene cuero triturado en pequeñas partes de aproximadamente media pulgada; cabe destacar que el agua evita que las fibras se precalienten en el proceso o terminen suspendidas en el aire, cayendo por gravedad al tanque de recepción. (Addie \& Karpik, 2011). Luego de que la mezcla (viruta de cuero-agua) se encuentra en el tanque de recepción, se transportada a través del refinador.

El refinador permite que las fibras se despliegan y obtengan una mayor superficie, aumentando significativamente el aglomerado de las fibras. Utilizando un refinador de discos de $450 \mathrm{~mm}$ de diámetro (el diámetro del disco está dimensionado en base a conservar el tamaño de la viruta que sale del molino), se rasga el cuero, se liberan las fibras y se reducen a una longitud máxima aproximada de 0.25 pulgadas $(6,35 \mathrm{~mm})$ (Addie \& Karpik, 2011); una vez refinada la viruta de cuero, está junto con el agua pasa al tanque de mezclado por propulsión del mismo refinador.

\subsection{Segunda Unidad - Mezclado y Moldeado}


La presente etapa abarca desde la recopilación de la viruta de cuero del proceso de curtido hasta la recepción y pesaje. La presente unidad tiene como objetivo adicionar a la mezcla resina, colorante y agua, y agitarla para su preparación para luego verterse en las cajas de desagüe y formar las planchas de cuero reconstituido.

Una vez que la mezcla se encuentra en el tanque de mezclado, se agrega $0.3 \mathrm{~kg}$ de fibras bicomponentes Cellobond ${ }^{\circledR}, 0.1 \mathrm{~kg}$ de fibra de poliéster sin recubrimiento Trevira, $0.16 \mathrm{~kg}$ de Resina Platamid H005 y 3.025 1 de agua por kilogramo de viruta utilizada.

Fibras bicomponentes Cellobond ${ }^{\circledR}(0.5$ pulgadas y 3 denier): sus propiedades permiten una fuerte unión entre las fibras, alta resistencia del producto final, características textiles de los no-tejidos, conservación del volumen y poros y una facilidad de procesamiento (Advasa, s.f.).

Fibra de poliéster Trevira CS (1,5 pulgadas y 15 denier): sus propiedades permiten alta resistencia, alto módulo, bajo encogimiento, estabilidad al calor y a la luz. (Vigneswaran et al., 2014).

Resina Platamid H005: sus propiedades permiten una buena adhesión a varios sustratos polares, así como una buena resistencia a la limpieza en seco y al lavado hasta $60^{\circ} \mathrm{C}$ (Campus®, 2019).

Agua: líquido que se agrega basado en la concentración de sólidos de la mezcla.

Posteriormente se mezcla todo a través de un sistema de agitación a una velocidad de aproximadamente 100 rpm debido al nivel de cizalladura que se requiere; el periodo a mezclarse será de 60 min considerando el tipo de tanque a utilizarse (tanque abierto). Una vez que la mezcla esta lista, es transportada mediante una bomba centrifuga al sistema de tuberías controlado por llaves de las cajas de desagüe. Cuando la mezcla es vertida a la caja (la cantidad de mezcla a verterse en cada caja depende del tamaño y grosor de la plancha que se desea fabricar; se filtra inicialmente por gravedad el agua sobrante (el filtrado se realiza por medio de una tela de tocuyo); mientras un operario empareja la mezcla a lo largo de toda la caja con una plancha de acero inoxidable. $\mathrm{Al}$ momento que la mezcla se ha distribuido y el agua se encuentra acumulada en el fondo de la caja, se activa la bomba de vacío de anillo líquido por 5 minutos con la finalidad de absorber el agua, extraer burbujas de aire y obtener una superficie homogénea; creando una plancha de fibra tendida al aire (Ávalos, 2013). El agua excedente del aglomerado representa un aproximado del 98\% del agua adicionada (Aramayo, 2015). Culminado el vacío, se retira el molde con la placa de cuero reconstituido y se apila; posteriormente se transporta los moldes a la etapa de prensado.

\subsection{Tercera Unidad - Prensado y Secado}

La presente unidad tiene como objetivo proporcionar a la plancha de cuero reconstituido su resistencia, espesor y humedad final. Esta unidad inicia con la operación de pre-secado, en la que los moldes con las planchas de cuero reconstituido ingresan al secador de bandejas para ser secadas por un periodo aproximado de 3 horas a una temperatura cercana a los $90^{\circ} \mathrm{C}$, evitando perjudicar las propiedades del cuero regenerado (Ávalos, 2013). Después de salir de la etapa de secado, las planchas de cuero reconstituido pasan a la operación de prensado, donde se desmoldan y apilan alternadamente con placas de acero inoxidable en grupos de 10, para posteriormente ser sometidas a una presión por una prensa hidráulica de alrededor de 50 TN durante al menos 10 minutos, con la finalidad de compactar, brindar una mayor resistencia al desgarre y el espesor final de la plancha. Una vez que el prensado ha concluido, se procede a separar las planchas de cuero reconstituido de las placas de acero inoxidable, para ser transportadas a la operación de rebajado, donde la rebajadora se encarga de brindar un grosor uniforme de acuerdo con especificaciones técnicas. Finalmente, una vez que las planchas han sido rebajadas, pasan a la etapa de corte donde se usa unas cuchillas para dar las dimensiones finales a la plancha.

\section{RESULTADOS}

De acuerdo a la evaluación desarrollada para el diseño de un proceso industrial para el aprovechamiento continuo de viruta de cuero, se alcanzaron los siguientes resultados: 
El informe del análisis a nivel laboratorio de los componentes de la viruta (Figura 1), permitió determinar la factibilidad de ser utilizada como materia prima para obtener planchas de cuero reconstituido. De acuerdo con los resultados obtenidos en los parámetros evaluados, se puede afirmar que:

Potencial de Hidrogeno: Se obtuvo un valor de 3.47, el cual se encuentra dentro del rango óptimo establecido, por lo cual no será necesario realizar un proceso previo para regular la basicidad.

Cromo Total: Se obtuvo un resultado de $4,93 \mathrm{mg} / \mathrm{kg}$, valor que se encuentra dentro del rango entre 3,5 y 5 $\mathrm{mg} / \mathrm{kg}$.

Humedad: Se obtuvo un porcentaje de humedad de $58.05 \%$, valor que se encuentra dentro del rango óptimo establecido (50 - 60\%); sin embargo, es necesario mantenerlo dentro del rango mediante la instalación de un almacén de ambiente controlado.

Grasas y aceites: Se obtuvo como resultado $1.25 \mathrm{mg} / \mathrm{kg}$ presente en la viruta de cuero, valor menor al límite máximo de $0,5 \%$, por lo cual no será necesario un proceso previo de aplicación de tensoactivos o desengrasantes industriales.

\begin{tabular}{|c|c|c|}
\hline \multicolumn{3}{|c|}{ INFORME DE ENSAYO N²512/2019.A } \\
\hline \multicolumn{3}{|c|}{ Razón Social: Pieles del Sur E.I.R.L. } \\
\hline \multicolumn{3}{|c|}{ Dirección: Mza. J Lote 3-B1 Parq. Industrial Rio Seco - Arequipa - Peru CMA:CAM1597/2019 } \\
\hline \multicolumn{3}{|c|}{$\begin{array}{l}\text { Muestra Id: } 16237-N^{2} \text { Muestra: } 2512 / 2019 \text { - Virutas de cuero/ Punto de toma de muestra: } \\
\text { Proceso Rebajado / Una (01) unidad } 1 \mathrm{~kg} \text { aprox. }\end{array}$} \\
\hline \multicolumn{2}{|c|}{ Fecha de Emisión: --- } & pción: 16/05/2019 \\
\hline \multicolumn{3}{|c|}{$\begin{array}{l}\text { Presentación: Bolsa de polietileno sellada } \\
\text { Condición de la muestra: Temperatura ambiente } \\
\text { Procedencia de la muestra: Proporcionada por el cliente } \\
\text { Fecha de inicio de análisis: 16/05/2019 }\end{array}$} \\
\hline \multicolumn{3}{|c|}{ Resultados Analíticos } \\
\hline \multicolumn{3}{|c|}{ Análisis } \\
\hline Análisis & Unidad & Resultado \\
\hline $\mathrm{Ph}$ & $\ldots$ & 3,47 \\
\hline Humedad-AOAC & $\%$ & 58,05 \\
\hline Cromo total & $\mathrm{mg} / \mathrm{Kg}$ & 4,93 \\
\hline Aceites y grasas & $\mathrm{mg} / \mathrm{Kg}$ & 1,25 \\
\hline \multicolumn{3}{|c|}{ Métodos de Análisis e Informaciones complementarias } \\
\hline Método de Análisis & \multicolumn{2}{|c|}{ Método de Referencia } \\
\hline $\mathrm{pH}+5$ ueros & \multicolumn{2}{|c|}{ EPA METHOD 9045D soll and waste $\mathrm{pH}$} \\
\hline Humedad-AOAC & \multicolumn{2}{|c|}{$\begin{array}{l}\text { AOAC 945. 15-1945, Loss in drying (moisture) in cereal } \\
\text { adjunets }\end{array}$} \\
\hline Cromo total - Fertilizantes & \multicolumn{2}{|c|}{ AOAC 965.09 Nutrient (Minor) in fertilizers } \\
\hline Aceites y grasas - Suelos & \multicolumn{2}{|c|}{$\begin{array}{l}\text { Epa SW-846 Method } 907 \text { 1b n-hexane extrantable } \\
\text { material (HEM) for sludge, sediment, and solid sampies }\end{array}$} \\
\hline
\end{tabular}

Figura 1: Informe de análisis de laboratorio de las virutas de cuero Fuente: INACAL (s.f)

Con respecto a la elección del artículo para poder determinar las características de las planchas de cuero, se preseleccionaron dos potenciales artículos, las Plantillas, y Cinturones. En base a la información levantada en el estado del arte, se decidió basar el diseño del proceso de acuerdo a las características técnicas del artículo Plantilla, expuestas a continuación en la Tabla 2: 
Tabla 2. Dimensiones de la plancha

Dimensiones de la

plancha de cuero a

elaborarse

\begin{tabular}{cc}
\multicolumn{2}{c}{ elaborarse } \\
\hline Largo & $1.4 \mathrm{~m}$ \\
Ancho & $1 \mathrm{~m}$ \\
Espesor & $2.5 \mathrm{~mm}$ \\
Fuente: Elaboración propia.
\end{tabular}

Una vez que se determinó el tipo de artículo a fabricar, se desarrolló un esquema del proceso de obtención de cuero reconstituido (Figura 2) donde se establecieron tres unidades con los siguientes componentes:

\subsection{Unidad 1 - Acondicionamiento de Materia Prima}

La unidad de acondicionamiento de materia prima se encuentra constituida por la balanza, el molino, el tanque de recepción, la bomba helicoidal y el refinador; los cuales son descritos a continuación.

Balanza: Se plantea utilizar una balanza de plataforma de marca JBC modelo plataforma de una capacidad de $300 \mathrm{~kg}$ con una precisión de $5 \mathrm{gr}$; comúnmente utilizada para aplicaciones industriales. Cuya función principal es medir la cantidad de viruta de cuero a ser procesada.

Molino: Se plantea utilizar un molino de cuchillas de marca IMAFER modelo PET, que tiene un rendimiento de $200 \mathrm{~kg}$ por hora y un tamiz con perforaciones de $12,7 \mathrm{~mm}$ de diámetro. El molino cuenta con una cámara de molienda, donde la materia prima pasa por las cuchillas y es cortada hasta que el material tenga el tamaño adecuado.

Tanque de recepción: Se propone usar un tanque de recepción cilíndrico de base cóncava con cuatro patas de soporte, con capacidad de 600 l. Este tanque está específicamente diseñado para recepcionar la viruta de cuero y agua proveniente del molino.

Refinador: Se plantea utilizar un refinador de marca Weifang modelo ZDP450, con una capacidad de 6 -20 TPD y discos de $450 \mathrm{~mm}$ de diámetro; cuya función es dar flexibilidad y abrir las fibrillas para aumentar los puntos de conexión.

\subsection{Unidad 2 - Mezclado y Moldeado}

La Unidad de mezclado y moldeado de planchas de cuero reconstituido se encuentra constituida por el tanque de mezclado, la bomba centrifuga, las tres cajas de desagüe y la bomba de vacío de anillo líquido; los cuales son descritos a continuación.

Tanque de mezclado: Se propone emplear un tanque de mezclado cilíndrico de base cóncava con cuatro patas de soporte, con una capacidad de 7431 y con un sistema de agitación. Este tanque está específicamente diseñado para mezclar viruta de cuero, agua, fibras bicomponentes, fibras de poliéster y resina.

Bomba centrífuga: Se necesita una bomba centrifuga de marca Hidrostal de tipo $\mathrm{S}$ en conjunto con un motor de marca Edipesa, de modelo D2.1/2S, con un caudal de 10 1/s y una potencia de 5 HP; cuya función es bombear líquidos con sólidos en suspensión y pulpa de papel de forma inatascable.

Cajas de desagüe: Se plantea utilizar tres cajas de desagüe que constan de los siguientes elementos: caja, 
tronco piramidal inverso, plancha perforada y tela de tocuyo. El objetivo de las cajas de desagüe es moldear la plancha, drenar el agua sobrante y extraer burbujas de aire, haciéndola más compacta.

Bomba de vacío de anillo líquido: Se sugiere usar una bomba de vacío de anillo líquido de marca SIHI modelo LPHX.45008.AB.AGE.OK.1, con una potencia de absorción de 3,9 KW y una presión de succión mínima de 33 mbar absoluto; cuya función es aspirar líquidos y gases formando un vacío.

\subsection{Unidad 3 - Prensado y Secado}

La Unidad de prensado y secado de planchas de cuero reconstituido se encuentra constituida por la prensa hidráulica, la rebajadora y la cadena aérea de secado; los cuales son descritos a continuación.

Secador de bandejas: Se requiere un secador de bandejas fabricado para la remoción de humedad de las bandejas (capacidad de 150 bandejas) de la cámara de secado a través de una fuente de calor para la evaporación del agua que se va incorporando al aire por medio de las salidas de aire a medida que transcurre el proceso de secado.

Prensa hidráulica: Se propone utilizar una prensa hidráulica, fabricada para aplicar presión sobre 10 planchas de cuero reconstituido al mismo tiempo, con una presión de $50 \mathrm{TN}$ a una carrera de $500 \mathrm{~mm} / 20 \mathrm{~s}$.

Rebajadora: Se sugiere emplear una rebajadora de marca RIZZI modelo RLA 10, con una capacidad de producción de 45 planchas por hora; cuya función es homogeneizar la superficie de la plancha de cuero.

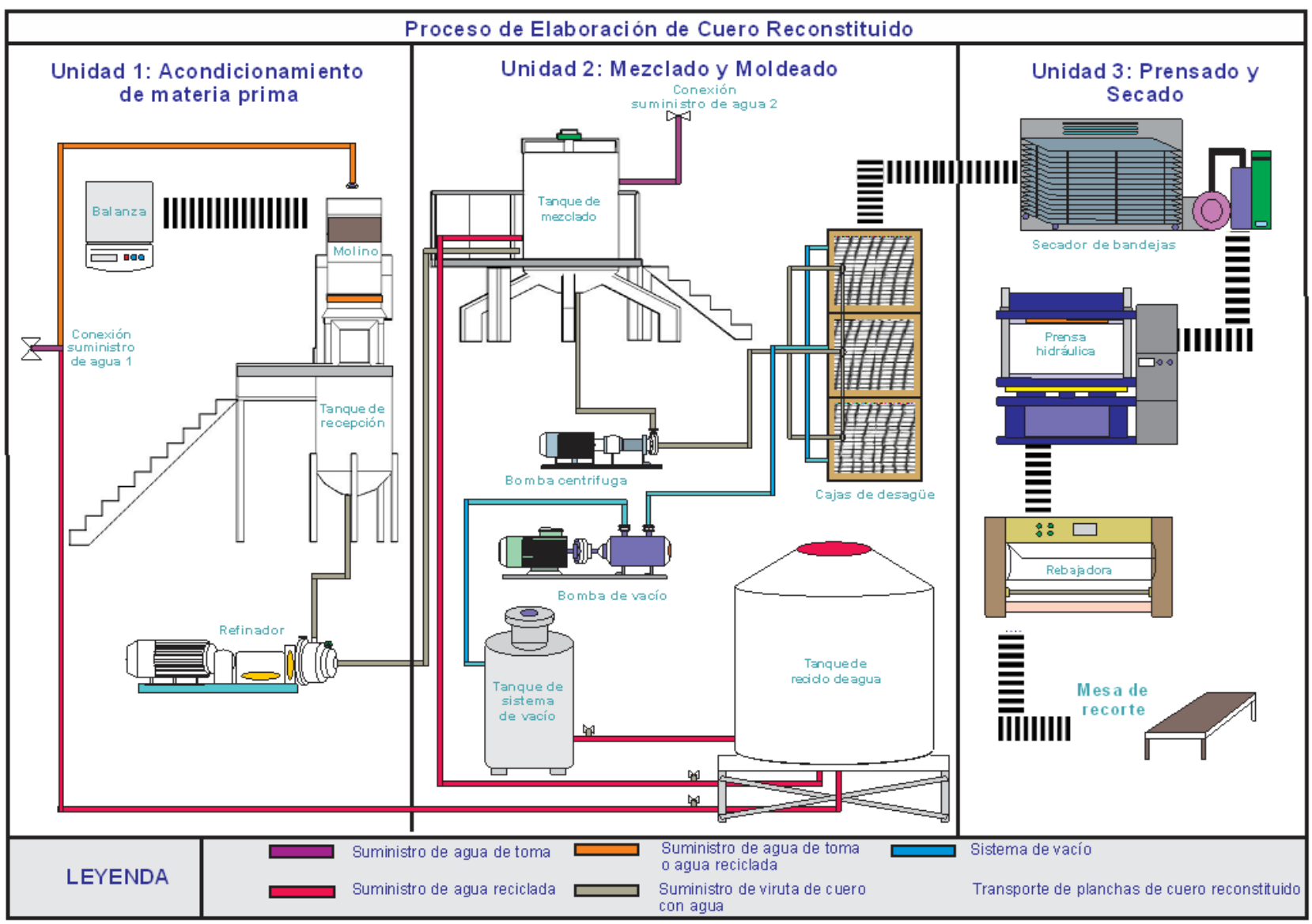

Figura 2: Esquema del proceso de elaboración de cuero reconstituido. 


\section{CONCLUSIONES}

- Se desarrolló un proceso productivo, a través del levantamiento del estado del arte, evidenciando metodologías de elaboración y operaciones básicas para obtener un cuero reconstituido, que tiene el potencial de asemejarse en tacto, color, acabado, olor, resistencia, flexibilidad y apariencia al cuero convencional.

- La revisión bibliográfica y el diseño del proceso demostró que la viruta tiene la potencialidad de ser empleada como materia prima para elaborar planchas de cuero reconstituido, empleando un proceso ecoamigable y estandarizado.

- Se ha cuantificado, caracterizado, establecido y validado los parámetros de control (potencial de hidrógeno, humedad, cromo total y grasas y aceites) del residuo sólido viruta de cuero, evidenciando su potencial para su aprovechamiento como materia prima en la elaboración de planchas de cuero reconstituido.

- Se recopilaron parámetros técnicos y de calidad de artículos elaborados a partir de cuero, se ponderaron los criterios y se seleccionó la plantilla como artículo para ser desarrollado.

- Se ha desarrollado una detallada metodología de tres unidades que se encargan de: 1. Acondicionar la materia prima 2. Mezclar y moldear la formulación 3. Prensar y secar la plancha de cuero reconstituido; asimismo se determinaron los equipos a utilizarse y sus especificaciones técnicas.

\section{REFERENCIAS}

Addie, B. A., \& Karpik, J. (2011). Reconstituted leather product and process. (Washington, DC. Estados Unidos de América. $\mathrm{N}^{\circ}$ de patente: US6264879B1). U.S. Patent and Trademark Office. https://patents.google.com/patent/US6264879B1/en

ADVANSA. (s.f.). Bicomponente (BiCo). Advanced Fibre Technology. https://www.advansa.com/es/aplicaciones-tecnicas/bicomponente/

Alberto, H. Retamar, J. Orué, S. Lacoste, A. \& Prez L. (2013). Virtudes de cuero obtención de un adhesivo como sustituto de materiales ureicos. http://www.aaiq.org.ar/SCongresos/docs/04_025/papers/07e/07e_1431_681.pdf

Aramayo, D. (2015). Obtención de cuero reconstituido a partir de residuos de cuero. Prezi. https://prezi.com/9lxy65we4zm/obtencion-de-cuero-reconstituido-a-partir-de-residuos-de-cue/

Avalos, V. (2013). Proceso productivo que permita la obtención de cuero regenerado de alta calidad (Informe final). Instituto de Investigación de la facultad de Ingeniería Química. http://hdl.handle.net/20.500.12952/1831

Azambuja, J. A. (2007). Couro reconstituido e processo de fabricação. (Brasil. $\mathrm{N}^{\circ}$ de patente: PI 0502929-5 A2). https://www.escavador.com/patentes/403954/couro-reconstituido-e-processo-de-fabricacao

Ananthasubramanian, M., Vigneswaran, C., \& Kandhavadivu, P. (2014). Bioprocessing of Textiles $\left(1^{\text {st }}\right.$ ed.) WPI Publising. https://doi.org/10.1201/9780367805524

CAMPUS (2019). Technical Data Sheet Resina Platamid H005. https://www.campusplastics.com/campus/pt/datasheet/Platamid\%C2\%AE+H+005/ARKEMA/179/316ae727

Cantera,C. \& Bertola,C. (2000) Valorización De Residuos Sólidos En La Industria Curtidora- Hidrólisis De Las "Virutas De Cromo". https://sistemamid.com/panel/uploads/biblioteca/2016-01-10_10-51-17131337.pdf 
Ley de Gestión Integral de Residuos Sólidos, Decreto Legislativo, $N^{\circ}$ 1278, 24 de Julio de 2004. https://sinia.minam.gob.pe/normas/ley-general-residuos-solidos

Medina K., Aguilar A. y Villegas J. (2018). Diseño de una propuesta de una planta industrial para el aprovechamiento de residuos sólidos del proceso de curtido. Nexo Revista Científica, 30 (2), pp. 02-14. https://doi.org/10.5377/nexo.v32i01.7989

Parker, E. T., \& Wayne, M. (1966). Reconstituted leather and method for producing it, (Washington, DC. Estados Unidos de América. $\mathrm{N}^{\circ}$ de patente: US3505169A). U.S. Patent and Trademark Office. https://patents.google.com/patent/US3505169A/en

Sánchez J. y Cortes R. (2016) Evaluación de la viruta de cuero generada en el proceso de rebajado para la fabricación de un aglomerado en San Benito. [Título profesional, Universidad de La Salle de Bogotá] https://ciencia.lasalle.edu.co/ing_ambiental_sanitaria/70/

\section{SEMBLANZA DE LOS AUTORES}

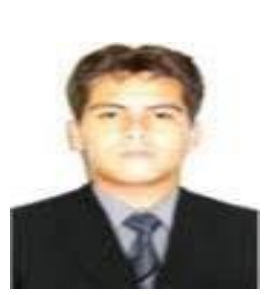

Jose A. Aguilar Franco: Obtuvo el grado de Ingeniero Industrial en la Universidad Católica San Pablo, Perú, donde actualmente es profesor titular, además de Ingeniero de Investigación del Programa deIniciación Científica, formulador de proyectos y Coordinador General del Institutode Energía y Medio Ambiente de la Universidad Católica San Pablo. Desarrolló sus estudios de maestría en la Pontificia Universidad Católica deRío de Janeiro (PUC - Río) y Universidad Nacional de San Agustín, Perú (UNSA). Ha desarrollado una estancia de investigación en Italia para Capacitación en Uso de Maquinarias Ecológicas. Cuenta con experiencia como asesor de Tesis, además trabaja como Formulador de Proyectos bajo la modalidad de concurso a nivel Nacional en proyectos de Investigación e Innovación convocadas por el Estado Peruano.

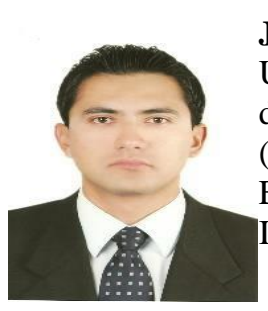

Jeanmarco Villegas Álvarez: Obtuvo el grado de Licenciado en Administración de Negocios en la Universidad Católica de San Pablo, Perú, donde actualmente es profesor titular. Desarrollo sus estudios de maestría en Administración de Empresas (MBA) en la Universidad Peruana de Ciencias Aplicadas (UPC). Cuenta con experiencia como especialista en dirección de entidades financieras por FUNCASEspaña, especialista en planeamiento de negocios y gestión estratégica y, Gestor de Proyectos de Innovación y Sociales.

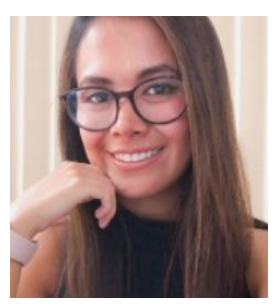

Alisson Rosario Alexandra Velando Moscoso: Obtuvo el grado de Ingeniero Industrial en la Universidad Católica San Pablo, Arequipa, Perú. Actualmente se desempeña como coordinadora de operaciones en la empresa DHL Supply Chain en Arequipa, Perú.

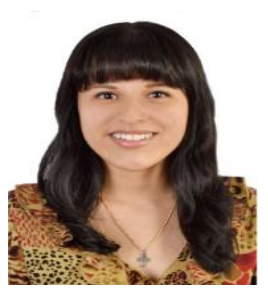

María Fernanda Zúñiga Núñez: Bachiller de la carrera profesional de Ingeniería Industrial en la Universidad Católica San Pablo, Arequipa, Perú. Actualmente viene desarrollando su tesis en el Instituto de Energía y Medio Ambiente (IEM) de la Universidad Católica San Pablo para obtener el título profesional de Ingeniero Industrial. 